Relations industrielles

Industrial Relations

\title{
La déclaration de principes de la FTQ
}

Volume 13, numéro 1, janvier 1958

URI : https://id.erudit.org/iderudit/1022473ar

DOI : https://doi.org/10.7202/1022473ar

Aller au sommaire du numéro

\section{Éditeur(s)}

Département des relations industrielles de l’Université Laval

\section{ISSN}

0034-379X (imprimé)

1703-8138 (numérique)

Découvrir la revue

Citer ce document

(1958). La déclaration de principes de la FTQ. Relations industrielles / Industrial Relations, 13(1), 99-102. https://doi.org/10.7202/1022473ar
Résumé de l'article

Voici le texte intégral de la « Déclaration de principes » de la Fédération des Travailleurs du Québec, telle qu'adoptée lors du deuxième congrès annuel régulier de cet organisme syndical affilié au Congrès du Travail du Canada, les 14,15 et 16 novembre 1957.
Tous droits réservés ( Département des relations industrielles de l’Université Laval, 1958
Ce document est protégé par la loi sur le droit d'auteur. L’utilisation des services d'Érudit (y compris la reproduction) est assujettie à sa politique d'utilisation que vous pouvez consulter en ligne.

https://apropos.erudit.org/fr/usagers/politique-dutilisation/ 
doit éclairer et animer»(S.S. Pie XII, ler juin 1941). Le laïc doit donc prendre ses responsabilités à la lumière des principes chrétiens enseignés par l'Eglise

«La sauvegarde du droit d'association. la recherche d'une saine organisation syndicale, dans le respect du droit naturel et des principes chrétiens par les patrons et les travailleurs, permettront d'espérer des relations patronales-ouvrières plus harmonieuses et mieux ordonnées.

«Nous tenons à attirer aussi votre attention sur la nécessité de relations harmonieuses entre les employeurs et les ouvriers de Notre diocèse. Ces rapports, comme Nous l'avons déjà signalé, en passant, doivent être empreints d'un désir profond et sincère de vérité, de justice et de charité. «L'Eglise appuie également tout ce qui contribue à rendre les rapports entre patrons et ouvriers plus humains, plus chrétiens et animés de confiance réciproque. La lutte des classes ne peut jamais être une fin sociale. Les discussions entre patrons et ouvriers doivent avoir pour objectif principal la concorde et la collaboration » (Pie XII, 11 mars 1951).

\section{But соммuN}

«En effet, seules des négociations franches et honnêtes pourront conduire à d'heureuses ententes entre le capital et le travail. L'exagération dans les revendications ou la fraude dans l'exposé des situations économiques conduisent tôt ou tard à la méfiance dans les relations patronales-ouvrières et préparent des luttes dont l'issue est dommageable à tous. «Dans le domaine économique, il y a communauté d'intérêts et d'activités, entre chefs d'entreprises et ouvriers. Méconnaître ce lien réciproque, travailler à le briser, ne peut être que le fait d'une prétention de despotisme aveugle et déraisonnable. Chefs d'entreprises et ouvriers ne sont pas antagonistes inconciliables, ils sont coopérateurs dans une oeuvre commune 》(Pie XII, 7 mai 1949).

\section{LA DECLARATION DE PRINCIPES DE LA FTQ}

Voici le texte intégral de la «Déclaration de principes» de la Fédération des Travailleurs du Québec, telle qu'adoptée lors du deuxième congrès annuel régulier de cet organisme syndical affilié au Congrès du Travail du Canada, les 14, 15 et 16 novembre 1957.

\section{EDUCATION}

La Fédération des travailleurs du Québec est consciente de la nécessité d'assurer la meilleure éducation possible à tous les citoyens de cette province, sans quoi ils ne pourront participer à la mise en valeur de ses ressources innombrables et les progrès techniques auxquels nous assistons présentement en feront de complets déclassés.

Elle réclame donc l'enseignement réellement et absolument gratuit à tous ses paliers: primaire, secondaire, universitaire et spécialisé. Elle soutient en outre que l'instruction devrait être obligatoire jusqu'à l'âge de 16 ans et qu'on devrait faire respecter avec la plus grande rigueur la loi qui en déciderait ainsi.

Elle reconnaît les droits fondamentaux de la province en matière d'éducation. Elle ne croit pas, cependant, que ce principe puisse permettre de négliger l'éducation. Ces droits comportent au contraire des devoirs graves dont le gouvernement de cette province doit s'acquitter de façon prioritaire. 
La Fédération affirme que pour remplir son rôle efficacement, l'enseignement doit être à l'abri de la politique de parti, ce qui ne veut pas dire qu'il ne comporte par l'éducation politique du futur citoyen. Nous réclamons donc la liberté académique la plus complète, en conformité avec la morale naturelle et nos principes démocratiques.

Nous réclamons, pour ceux qui consacrent leur vie à l'enseignement, des salaires qui les mettent à l'abri des soucis matériels et qui leur assurent le prestige social auquel ils ont droit et qui est essentiel à la réussite de leur mission. Nous ne voulons pas que l'éducation de nos enfants soit confiée à des laissés pour compte, humiliés et soumis, qui ne sauraient en faire les citoyens libres et fiers dont notre société a besoin.

Nous réclamons l'établissement d'un Conseil supérieur de l'éducation vraiment représentatif de tous les groupes sociaux - y compris le mouvement syndical - et éducationnels. Ce Conseil aurait la responsabilité des programmes et du budget de l'enseignement; mis à l'abri de la petite politique de parti, il devrait s'entourer des plus grandes compétences.

\section{SÉCURITÉ SOCIALE}

La Fédération des travailleurs du Québec affirme que cette province peut mettre en valeur des ressources naturelles si grandes et s'industrialiser à un tel point qu'elle n'a pas d'excuse à ne pas mettre sur pied un programme complet de sécurité sociale, condition essentielle de la survie de notre régime démocratique. Elle demande donc la mise en oeuvre d'un tel programme afin de faire disparaître la pauvreté et l'insécurité d'une province aussi riche, et d'y substituer la justice sociale à la charité publique, la charité ne devant subsister qu'à titre de vertu individuelle.

Un tel programme doit assurer aux vieillards, aux personnes handicapées, aux veuves et aux orphelins un niveau de vie décent qui leur permettra surtout de recouvrer le sens de la dignité personnelle.

La Fédération réclame en outre la conclusion immédiate d'une entente entre les gouvernements national et provinciai pour la mise en application d'un programme complet d'assurance-santé, dont l'administration relèvera de la province. Elle ne croit pas qu'un tel accord, consenti librement par le gouvernement provincial, soit incompatible avec les dispositions de l'Acte de l'Amérique du Nord, ni qu'elle lèse en rien le principe de lautonomie provinciale, qui n'a pas été compromise jusqu'ici par les pensions de vieillesse et les allocations familiales.

\section{LÉGISLATION OUVRIÈRE}

La Fédération des travailleurs du Québec, consciente de sa responsabilité dans l'obtention d'une législation juste qui protège les droits des syndicats et des salariés qui les composent, de même que les droits des travailleurs qui veulent se grouper en syndicats, réclame un code provincial du travail.

Aucune loi du travail ne sera acceptable aux travailleurs à moins qu'elle ne consacre intégralement trois droits fondamentaux:

1) DROIT D'ASSOCIATION - Ce droit est naturel et aucune loi ne doit le détruire, le compromettre ou en restreindre l'exercice. Contrairement à ce que prévoit ou permet la loi actuelle, ce droit doit être reconnu, indépendamment de tout autre facteur, à tout syndicat grounant la majorité d'un groupe de travailleurs. La reconnaissance officielle d'un syndicat ne doit jamais être subordonnée aux actes faits par ce syndicat et le retrait de celleci ne doit jamais servir de sanction contre un syndicat qui reste majoritaire. 
De plus, la loi doit prévoir des sanctions sévères et efficaces contre toute personne ou tout groupe de personnes qui, par des menaces, des congédiements, la corruption, le chantage ou la création de syndicats de boutique, entrave le libre exercice du droit d'association.

2) DROIT A LA NEGOCIATION COLLECTIVE - La Fédération des travailleurs du Québec affirme que les travailleurs ont droit à une législation efficace qui garantisse la possibilité de négociations de bonne foi entre tout groupe syndical majoritaire et un ou des patrons. Les procédures de conciliation et de médiation, pour conserver leur raison d'être, doivent être confiées à des personnes compétentes, socialement éclairées et à l'abri de toute influence ou ingérence politiques. Dans le contexte actuel, ces procédures provoquent une suspicion instinctive dans le mouvement syndical.

3) DROIT A LA GREVE - La Fédération des travailleurs du Québec est consciente des conséquences économiques qui peuvent résulter du droit de grève. Ses syndicats affiliés n'en ont d'ailleurs jamais usé qu'avec discernement. Cependant, ce droit reste fondamental et la grève demeure, en définitive, la seule arme des travailleurs. La Fédération réprouve donc toute mesure ou loi qui en prohibe ou en restreint l'exercice.

Seuls ceux à qui ce droit appartient peuvent décider d'en user ou de n'en pas user. Assurée qu'en toutes circonstances ceux qui devront exercer ce droit sauront sauvegarder la sécurité et le bien-être des citoyens en général, la Fédération réclame le droit à la grève pour tous les travailleurs, quels que soient l'industrie, les services publics ou tout autre service pour lesquels ils travaillent.

\section{Ressources Naturelles}

La province de Québec possède des ressources naturelles inestimables qui constituent la plus grande richesse de sa population. Ces ressources et ces richesses appartiennent de droit, et en premier lieu, aux citoyens de cette province. Il est normal qu'étant au départ notre propriété commune, elles soient exploitées d'abord en vue du bien commun.

La Fédération des travailleurs du Québec croit donc que les citoyens de cette province doivent pouvoir participer à la mise en valeur et à l'exploitation de ces richesses. Ils doivent en tirer des avantages économiques de nature à relever leur standard de vie, à leur assurer la sécurité sociale, le plein emploi, l'éducation gratuite, ainsi que des dégrèvements fiscaux proportionnés à leur état de fortune.

La Fédération croit que si, en certains cas, l'exploitation de nos ressources nécessite l'apport de capitaux étrangers, une part importante de la gérance de l'entreprise et de ses profits doit rester entre les mains des citoyens de cette province. Aux travailleurs engagés dans l'exploitation de ces richesses, le gouvernement se doit alors d'assurer le libre exercice du droit d'association et de grève, ainsi que des salaires au moins égaux à ceux qui sont payés ailleurs dans des industries semblables. Il doit veiller surtout à ce que les droits fondamentaux du citoyen soient sauvegardés dans les nouvelles villes isolées ou à demi-fermées.

La Fédération des travailleurs du Québec croit que toute concession de nos ressources naturelles à l'entreprise privée doit être conditionnelle et que l'entreprise doit rester sujette à la nationalisation si les droits ou les intérêts des citoyens sont lésés, ou si ces derniers jugent qu'ils peuvent en tirer un meilleur parti autrement.

\section{Education et action politique}

L'influence du politique sur l'économique et le social s'accroît sans cesse et elle est d'autant plus évidente. dans la province de Québec qu'elle s'exerce constamment 
de façon préjudiciable pour les meilleurs intérêts des travailleurs. L'action politique n'est pas seulement l'aboutissement de l'action syndicale, pour nous; elle est la condition même de la survie de notre mouvement.

Les travailleurs syndiqués sont des citoyens comme les autres. Ils doivent donc s'intéresser aux lois qui gouvernent leur vie au sein de la société comme à celles qui régissent leur vie à l'usine.

Les rudes expériences d'un passé récent ont donné de la maturité à notre mouvement et l'ont rendu conscient du rôle politique qu'il doit jouer, dans le cadre de notre système démocratique parlementaire, aussi bien par le vote de ses membres que par les pressions collectives de ses divers organismes.

La Fédération des travailleurs du Québec a acquis la conviction que l'expansion du syndicalisme et l'avènement de la sécurité sociale, si nécessaire à la famille du travailleur, ne pourront jamais être assurés par les forces politiques en place. Aussi ne voit-elle de solution que dans l'éducation et l'action politiques, l'une ne pouvant aller sans l'autre.

Elle entend donc accélérer l'éducation politique de ses membres, étudier les structures politiques actuelles, y chercher les correctifs nécessaires et, pour voir à leur application, inciter ses membres à l'action politique directe.

A ce propos, la Fédération étudie les solutions qui s'offrent à elle et se propose d'en inventer qui lui soient propres, au besoin. Elle se propose de réexaminer constamment la situation politique à la lumière des facteurs nouveaux qui peuvent se présenter à son attention, et de dresser ainsi un programme d'action directe à l'intention de ses membres. Dans l'immédiat, elle voit la nécessité de donner son appui au parti politique qui hui fournira les meilleures garanties en travaillant sérieusement et de façon constante dans l'intérêt de la classe laborieuse.

La Fédération entend collaborer en outre avec tous les groupes de citoyens qui s'intéressent activement à la régénération de la démocratie politique sur le plan provincial, afin:

a) QUE chaque citoyen puisse jouir librement de son droit de vote;

b) QU'aucune classe, ni aucun groupe n'exerce sur le plan électoral une puissance incompatible avec sa force numérique;

c) QUE les partis politiques et les candidats soient réellement les représentants de ceux qui les élisent et non pas les valets de certaines forces occultes ou d'une autocratie qui confine à la dictature;

d) QUE la corruption, les menaces, le chantage, les promesses fallacieuses et le gangstérisme disparaissent des campagnes électorales et que les candidats soient tenus de dévoiler entièrement la source de l'argent qu'ils y dépensent;

e) QUE la carte électorale soit revisée de façon à assurer une représentation équitable à tous les citoyens;

f) QUE la loi électorale soit modifiée de facon à assurer le même traitement à tous les candidats et à leur fournir la même protection contre les fraudes électorales;

g) QUE les citoyens aient accès à une information complète et impartiale sur les travaux parlementaires, par la publication d'un journal des débats (Hansard);

h) QUE l'opposition démocratique, parlementaire ou extra-parlementaire, puisse discuter et critiquer librement les gestes du gouvernement, sans faire lobjet de menaces, de chantage ni de représailles. 